\title{
Space-Charge Limitation of a Femtosecond Photoinjector
}

\author{
Hiroko Yamamoto, Hiroaki Hamabe, Shintaro Sone, \\ Soichiro Yamaguchi, and Makoto R. Asakawa
}

Department of Pure and Applied Physics, Faculty of Engineering Science, Kansai University, 3-3-35 Yamate-cho, Suita-shi, Osaka 564-8680, Japan

Correspondence should be addressed to Makoto R. Asakawa, asakawa@kansai-u.ac.jp

Received 31 May 2011; Revised 23 July 2011; Accepted 2 August 2011

Academic Editor: Marco Zangrando

Copyright ( 2011 Hiroko Yamamoto et al. This is an open access article distributed under the Creative Commons Attribution License, which permits unrestricted use, distribution, and reproduction in any medium, provided the original work is properly cited.

Experimental investigation of a compact $40-\mathrm{kV}$ diode-type photoelectric DC gun driven by $100 \mathrm{fs}$ laser pulses revealed that the space-charge-limited current density could exceed $30 \mathrm{kA} / \mathrm{cm}^{2}$ and that the density increased linearly with the accelerating voltage. We explained these important properties by the balance between the cathode surface field and the field produced by sheet-like electron bunches near the cathode surface. Our simple physical model agreed well with the experimental results.

\section{Introduction}

Electron guns are basic tools for commercial engineering and state-of-the-art science. Many types of guns have been developed and are still being investigated for wide-ranging application fields, such as electron beam welding, lithography, and X-ray generation. Among them, electron guns using a photocathode (called photoinjectors) are excellent candidates for generating high-brightness electron bunches [1]. Photoinjectors are classified into two types according to the method used for generation of the acceleration field: RF photoinjectors $[2,3]$ and DC photoinjectors $[4,5]$. Both types are primarily being developed for the next generation of accelerators for use in free-electron lasers and high-energy physics because they can produce high-charge $(\sim 1 \mathrm{nC})$ electron bunches with perfectly controlled timing. By virtue of its structural simplicity, the DC photoinjector is being investigated as a compact electron bunch source in other research fields such as ultrafast electron microscopy [6]. In our research, a $40-\mathrm{kV}$ DC photoinjector is being developed as a compact electron gun to produce ultrashort, high-charge electron bunches. The bunches will be used to generate intense coherent terahertz light waves using the coherent radiation effect of bunches whose length is shorter than the radiation wavelength $[7,8]$. To date, most research on
DC photoinjectors was performed using nanosecond lasers [9] and picosecond lasers $[6,10,11]$. In these studies, the space-charge limitation followed the Child-Langmuir law; the maximum current density was proportional to $V_{\mathrm{KA}}{ }^{1.5}$ and was limited to the order of $10 \mathrm{~A} / \mathrm{cm}^{2}$. The space-charge limitation of such DC photoinjectors was the same as that of conventional electron guns using thermionic cathode. This is because the electron bunch length along the propagating direction was greater than or comparable to the gap distance between the cathode electrode and the anode electrode: the A-K gap was plugged with electrons, similar to that in the thermionic electron gun. With femtosecond lasers, however, the situation changes. The length of the electron bunch is less than $100 \mu \mathrm{m}$ because of the short duration of photoemission; thus, electrons occupy only a small part of the A-K gap. This type of gun can produce much denser electron bunches beyond the limitation of the Child-Langmuir law. We obtained a $744 \mathrm{pC}$ bunch from our compact 40-kV DC photoinjector driven by a $100 \mathrm{fs}$ laser. This charge is comparable to that obtained from more energetic RF/DC photoinjectors for high-energy physics; amazingly, the corresponding current density exceeds $30 \mathrm{kA} / \mathrm{cm}^{2}$, which is 4000 times greater than the Child-Langmuir limit. In this paper, we describe our experimental results and a physical model that explains the space-charge limitation for such short-pulse photoinjectors. 


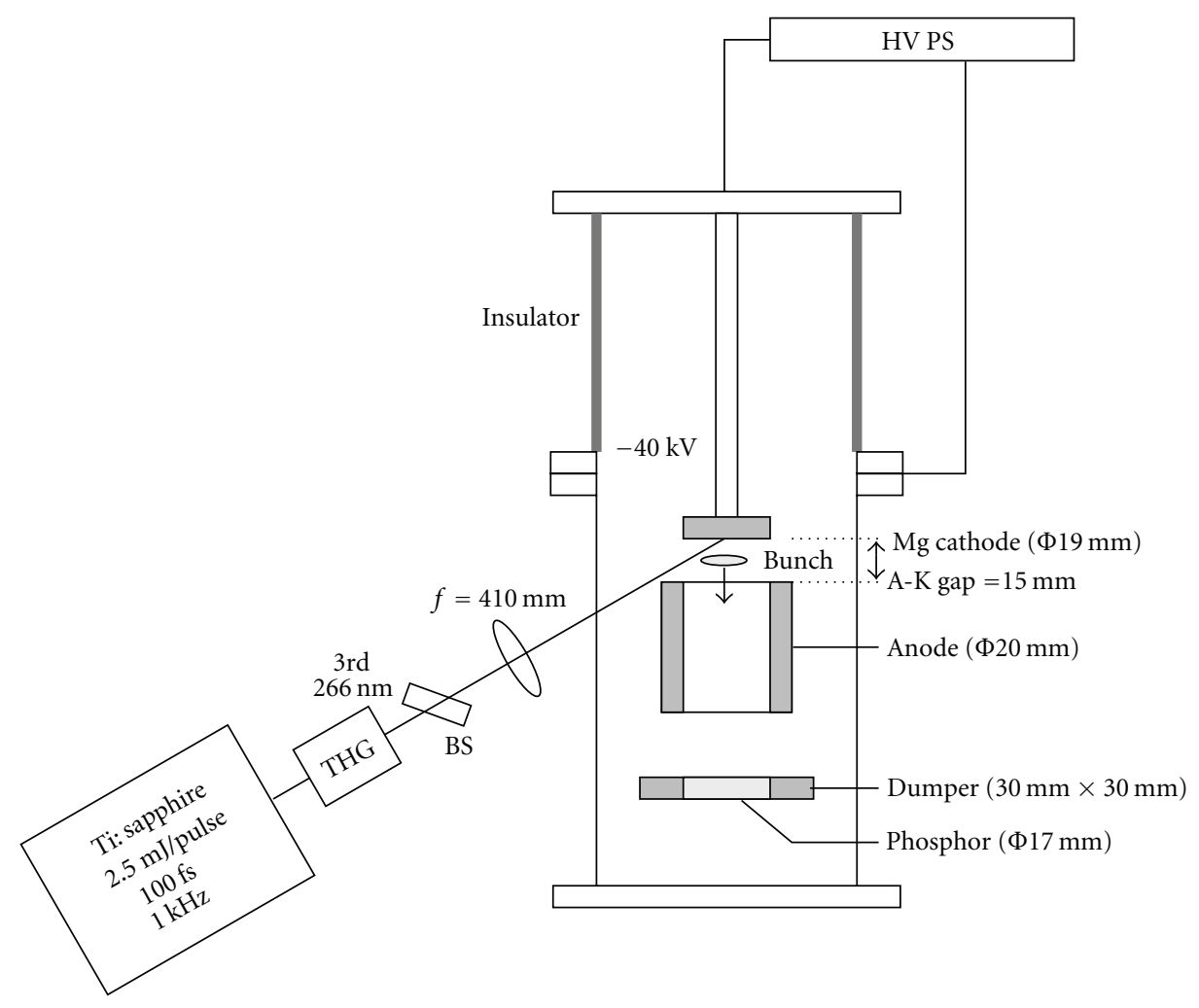

FIGURE 1: Experimental apparatus. THG: third-harmonic generator, HV PS: $40 \mathrm{kV}$ high-voltage power supply.

\section{Experimental Apparatus}

Figure 1 shows a schematic view of the compact $40-\mathrm{kV}$ DC photoinjector. The exterior of the injector was $35 \mathrm{~cm}$ in length and $11 \mathrm{~cm}$ in diameter, which is very compact compared to DC photoinjectors developed for next-generation accelerators. A frequency-tripled Ti:sapphire laser beam $(\lambda=$ $266 \mathrm{~nm}, h v=4.7 \mathrm{eV}$ ) irradiated a magnesium photocathode (work function $=3.64 \mathrm{eV}$ ) installed in the diode-type DC gun to produce photoelectrons. The repetition rate of the laser was $1 \mathrm{kHz}$, and its pulse width fluctuated slightly in 95$105 \mathrm{fs}$ range. The laser beam was focused by a lens placed outside the vacuum vessel and irradiated the cathode, which was $20 \mathrm{~mm}$ in diameter, at an incident angle of $60^{\circ}$. The area of the elliptical laser spot was $\pi 1.5 \times 5=24 \mathrm{~mm}^{2}$. The cathode was connected to a high-voltage DC power supply with a maximum voltage of $-40 \mathrm{kV}$. The DC electric field accelerated the photoelectrons toward the anode electrode and a dumper electrode placed immediately behind the anode electrode, at which a device for generating coherent radiation, such as a metallic grating or dielectric slabs, will be installed. A phosphor plate $17 \mathrm{~mm}$ in diameter was installed in the dumper electrode to observe the charge density profile. The anode-cathode gap was $15 \mathrm{~mm}$. The anode electrode was a thin-wall cylinder $5 \mathrm{~cm}$ in length with an inner diameter of $20 \mathrm{~mm}$ and an outer diameter of $24 \mathrm{~mm}$. Thus, some of the accelerated electrons hit the anode electrode, and residual electrons hit the dumper electrode. The electrodes were connected to the ground through $50 \Omega$ shunt resistors; the charges flowing through the resistors were monitored by a 10 Gsamples/s oscilloscope.

Figure 2(a) shows a typical time trace of the anode shunt resistor signal. A sharp negative spike at $90 \mathrm{~ns}$ on the abscissa indicates the current flowing in the anode resistor. Because of impedance mismatch, the spike is followed by a ringing signal, which vanishes with time integration. The time constants of the anode and dumper charge monitors were approximately $5 \mathrm{~ns}$ and were determined primarily by the stray capacitance between the electrodes and the vacuum vessel. The bunch charges were evaluated by integrating the resistor current. The laser energy was varied from 8 to $230 \mu \mathrm{J} / \mathrm{pulse}$ by changing the incident angle of the laser beam with a multilayered beam splitter. A laser spot size of $24 \mathrm{~mm}^{2}$ was carefully chosen to avoid laser ablation of the cathode for all experimental conditions. Figure 2(b) shows the anode shunt resistor signal when the laser irradiance was extremely intense. The accelerating voltage was reduced to $3 \mathrm{kV}$ in this case. A slowly varying negative signal $40 \mathrm{~ns}$ in duration appeared between 95 and $135 \mathrm{~ns}$ on the abscissa, following the electron bunch spike. This undesirable current may come from plasma excited at the cathode surface. With a higher accelerating voltage, this plasma current increased significantly, eventually short-circuiting the electron gun. All experiments were performed under a pressure of $4 \times 10^{-6} \mathrm{~Pa}$, and the stability of the bunch charge was within $4 \%$ for each incident laser pulse energy. 


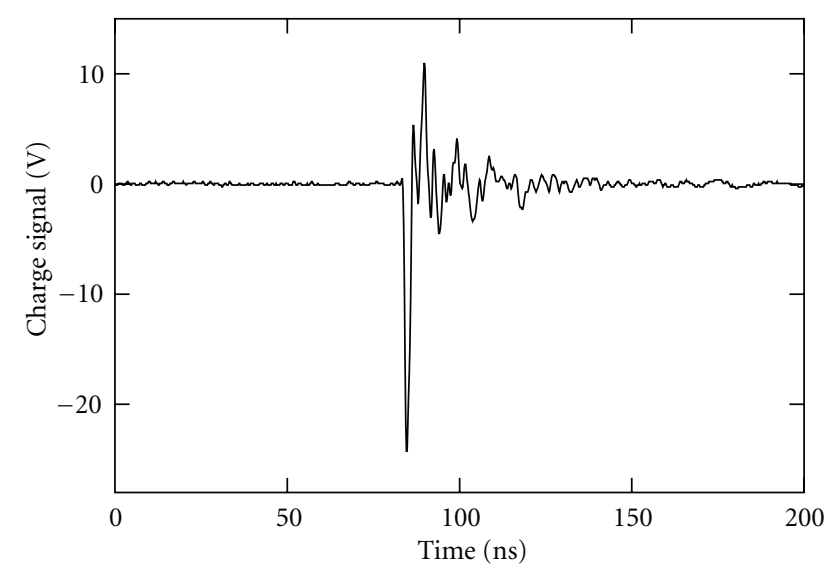

(a)

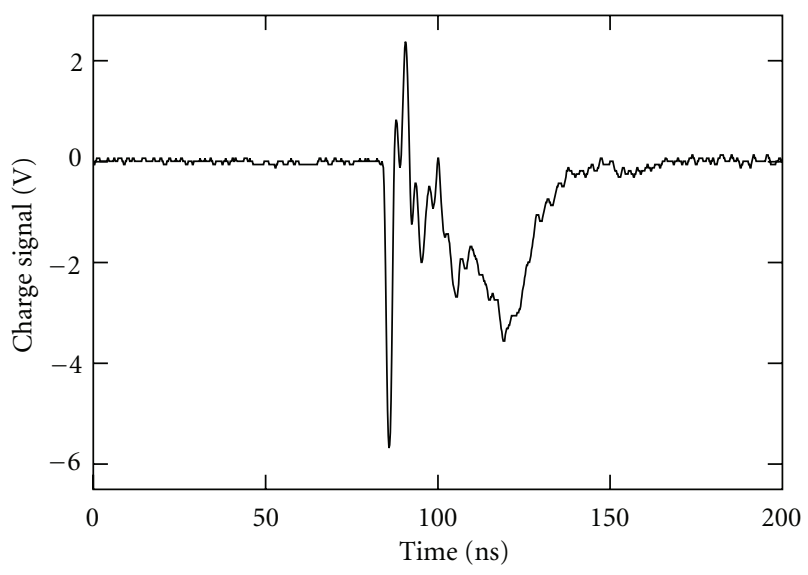

(b)

FIGURE 2: Time trace of the charge monitor: (a) typical waveform of the anode charge monitor at an accelerating voltage of $40 \mathrm{kV}$, (b) waveform of the anode charge monitor when the laser irradiance was so intense that laser ablation could occur.

\section{Experimental Results}

With an accelerating voltage of $40 \mathrm{kV}$ and a laser irradiance of $205 \mu \mathrm{J}$, maximum bunch charge of $744 \mathrm{pC}$ was observed without significant plasma production. The corresponding current density exceeded $30 \mathrm{kA} / \mathrm{cm}^{2}$. This result is the most attractive aspect of the femtosecond photoinjector compared to injectors driven by a longer laser pulse. This current density is several thousand times the saturation density evaluated by the Child-Langmuir law.

Figure 3 shows the bunch charge as a function of the laser energy with different accelerating voltages. When the accelerating voltage was $10 \mathrm{kV}$, the bunch charge increased from 0 to $120 \mathrm{pC}$ as the laser energy increased from 0 to $50 \mu \mathrm{J}$. At $95 \mu \mathrm{J}$, the bunch charge leveled off, and then the charge remained almost constant at approximately $150 \mathrm{pC}$ for a laser irradiance up to $210 \mu \mathrm{J}$. That is, the photoinjector was saturated at an irradiance greater than $95 \mu \mathrm{J}$. The same tendency appeared with an accelerating voltage of $30 \mathrm{kV}$ : the bunch charge increased from 0 to $280 \mathrm{pC}$ as the laser energy increased from 0 to $100 \mu \mathrm{J}$, whereas for higher laser

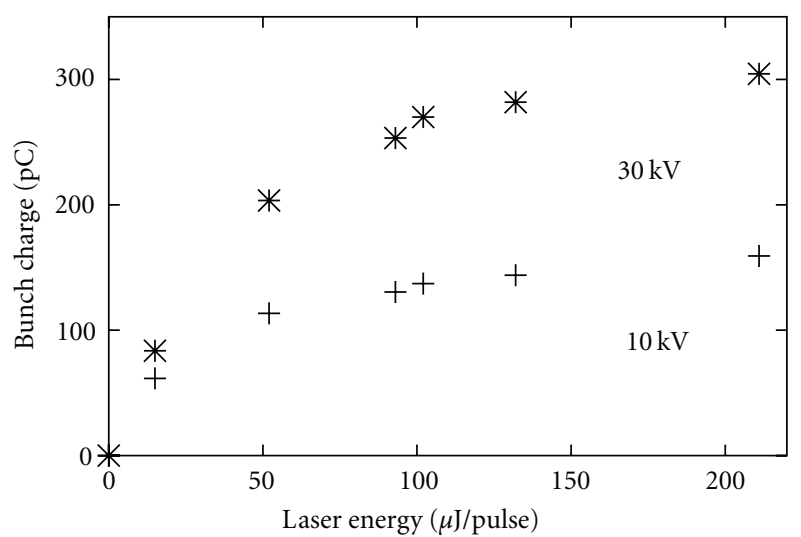

Figure 3: Bunch charge versus laser energy. At high laser energy, the bunch charge is saturated.

irradiance, the charge remained almost constant, and the saturation charge increased to $300 \mathrm{pC}$. Increase in the laser energy indicates increase in the number of photons causing photoemission, which leads to the increase in the number of photoelectrons produced at the cathode surface. Therefore, the observed saturation behavior indicates that the number of electrons to be accelerated is limited by the accelerating voltage. This type of saturation phenomenon is understood as the so-called space-charge-limited. Closely observing the saturation regime, we find that the bunch charge increases very slightly with the laser energy. This behavior may originate in the nonuniform flux distribution of the incident laser: because of the Gaussian spatial profile of the laser intensity, the central part of the electron bunch reached saturation first, and then the increase in the laser energy broadened the saturated region toward the bunch's periphery.

According to the discussion of the space-charge-limited above, the saturation effect might be observed more clearly at a lower accelerating voltage. Therefore, we investigated in detail the space-charge limitation charge at an accelerating voltage ranging from 0 to $16 \mathrm{kV}$. Figure 4 shows the bunch charge as a function of the accelerating voltage with laser irradiances of 69 and $130 \mu \mathrm{J}$. The dotted lines denote the predictions of the physical models described in the next section. At a laser energy of $130 \mu$, the bunch charge increased linearly from 0 to $85 \mathrm{pC}$ as the accelerating voltage increased from 0 to $5 \mathrm{kV}$. This dependency violates the Child-Langmuir law. At a higher laser irradiance, the rate of increase of the bunch charge gradually declined as the accelerating voltage increased. At a laser energy of $69 \mu \mathrm{J}$, the same tendency appeared: the bunch charge increased linearly from $0 \mathrm{pC}$ to $60 \mathrm{pC}$ as the accelerating voltage increased from 0 to $4 \mathrm{kV}$. From these results, we conclude that the spacecharge limitation charge of the short-pulse DC photoinjector depends linearly on the accelerating voltage. Decrease in the rate of increase of the bunch charge observed at an accelerating voltage greater than $5 \mathrm{kV}$ is also explained by the Gaussian spatial profile of the incident laser, as follows. Increase in the accelerating voltage reduces the saturated emission region of the bunch inward from its periphery, where the number ofphotoelectrons is much smaller than 


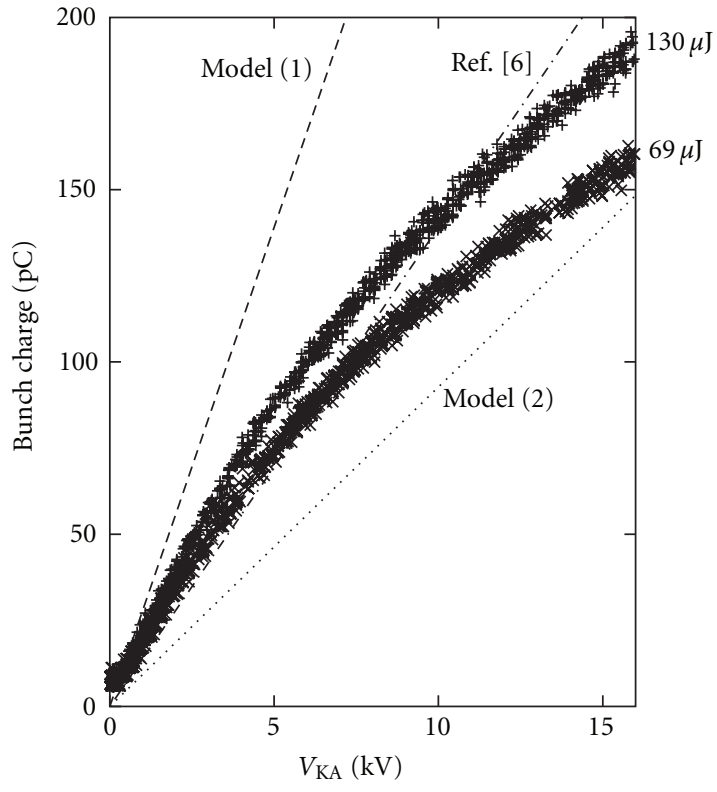

Figure 4: Bunch charge versus accelerating voltage. Dotted lines denote the predictions of our physical model; dash-dotted line represents the criterion described in [6]. Space-charge-limited charge increases linearly as the accelerating voltage increases from 0 to $5 \mathrm{kV}$.

that in the central region. Although the number of electrons emitted in the central saturated region increased linearly with the accelerating voltage, the number of electrons from the periphery remained constant because this region is in the socalled emission limit condition. Thus, the rate of increase of the total charge declined.

\section{Modeling the Space-Charge Limitation}

Next, we build a physical model to describe the space-charge limitation of the femtosecond photoinjector. The model should explain the extremely high saturation density and the linear dependence of the bunch charge on the accelerating voltage.

First, application of the accelerating voltage $V_{\mathrm{KA}}$ induces the surface charge density $\sigma_{\text {surf }}$ on the cathode. This charge increases with the electric field at the cathode surface given by $E_{\text {surf }}=V_{\mathrm{KA}} / d$. The relationship between the charge density and the electric field is given as $\sigma_{\text {surf }}=\varepsilon_{0} V_{\mathrm{KA}} / d$, where $d$ is the gap between the anode and cathode electrodes. Irradiation of laser photons onto the cathode causes photoemission. Owing to the short period of photoemission, the emitted electrons form a sheet-like bunch with the surface density $\sigma_{\text {photo }}$. Here we consider the charge density of the cathode surface immediately after photoemission. We have two cases.

(1) Surface Density Remains Constant at $\sigma_{\text {surf. }}$ Figure 5 shows relationship between the electric field produced by an electron bunch $E_{\text {photo }}$ and the field at the cathode surface $E_{\text {surf }}$. When $E_{\text {photo }}$ increases to $E_{\text {surf }}$, that is, $E_{\text {photo }}=E_{\text {surf }}$, the accelerating field on the cathode surface vanishes. Analogous to the saturation under the Child-Langmuir law, the charge

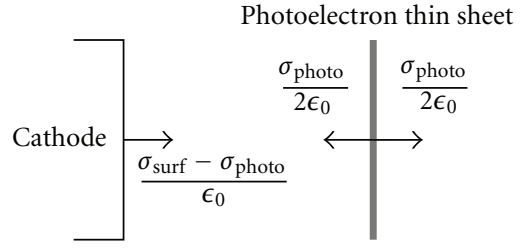

FIGURE 5: Electric fields produced by the cathode surface charge and the sheet-like electron bunch.

density $\sigma_{\text {photo }}$ under this condition is the saturation charge density because no more photoelectrons on the cathode surface are accelerated toward the anode electrode. Rewriting this condition, we have

$$
\frac{\sigma_{\text {surf }}}{\varepsilon_{0}}=\frac{\sigma_{\text {photo }}}{2 \varepsilon_{0}},
$$

where the right-hand side of the equation is the apparent form of the field produced by the sheet-like bunch. The space-charge-limited bunch density, therefore, is

$$
\sigma_{\text {photo }}=2 \varepsilon_{0} \frac{V_{\mathrm{KA}}}{d} .
$$

Multiplying the photoemission area by this density yields the saturation charge. This criterion is shown in Figure 4 as the upper dotted line.

(2) Surface Density Decreases to $\sigma_{\text {surf }}-\sigma_{\text {photo }}$. Considering the velocity of electrons in metals is in the order of $\sim \mathrm{cm} / \mathrm{s}$, no electrons may diffuse into the photoemission area during the very short (100 fs) photoemission process. Thus, the cathode surface charge density drops. Under this condition, we have

$$
\frac{\sigma_{\text {surf }}-\sigma_{\text {photo }}}{\varepsilon_{0}}=\frac{\sigma_{\text {photo }}}{2 \varepsilon_{0}} .
$$

Then, the space-charge limitation density is

$$
\sigma_{\text {photo }}=\frac{2}{3} \varepsilon_{0} \frac{V_{\mathrm{KA}}}{d} .
$$

This criterion is also shown in Figure 4 as the lower dotted line.

The experimental results clearly lie between these criteria. This suggests that the cathode surface charge is partially compensated during the $100 \mathrm{fs}$ long photoemission process. In addition to our approximate model, the criterion $\sigma_{\text {photo }}=$ $\varepsilon_{0} V_{\mathrm{KA}} / d$ described in [6], which is based on the virtual cathode formation, also fits well with our experimental results. This criterion is shown in Figure 4 as the dash-dotted line lying between our criteria. Our study supports this earlier research in which the injector could not be operated in the saturation regime. For a more precise comparison, the photoemission area and the charge density profile of the bunch should be measured accurately.

\section{Conclusion}

We investigated the performance of a compact $40-\mathrm{kV}$ DC photoinjector driven by ultrashort laser pulses in 
the saturation regime. The photoinjector could produce intense electron bunches with a current density greater than $30 \mathrm{kA} / \mathrm{cm}^{2}$. This property was explained well by the balance between the field of the cathode surface charge and that of the sheet-like electron bunch. However, it is readily expected that such intense bunches suffer a strong self-field, which increases their length along the propagation direction and enlarges their radial size. In fact, we observed a large radial divergence of bunches when the injector was operated in the saturation regime: the phosphor plate fluoresced uniformly over its entire area. This defect is the main reason that researchers avoid driving DC photoinjectors by ultrashort laser pulses, even though this is the most direct method to produce intense short electron bunches.

We are investigating the space-charge effect on the bunch volume to clarify whether the ultrashort-pulse DC photoinjector is valuable as an advanced compact electron bunch source for coherent radiation generation. The most interesting and critical issue is the increasing effect of the space charge on the bunch length. A time-domain spectroscopy system for measuring the waveform of the radiation emitted from the electron bunches is now under development using the photocathode driving laser. We hope that this development will clarify the relationship between bunch charge and bunch length.

\section{Acknowledgment}

This research was partially supported by the Ministry of Education, Science, Sports and Culture, Grant-in-Aid for Scientific Research, no. 21540514, 2011.

\section{References}

[1] C. Hernandez-Garcia, M. L. Stutzman, and P. G. O'Shea, "Electron sources for accelerators," Physics Today, vol. 61, no. 2, pp. 44-49, 2008.

[2] Y. E. Sun, J. W. Lewellen, and D. W. Feldman, "Photothermal cathode measurements at the advanced photon source," in Proceedings of the International Linear Accelerator Conference (LINAC '06), Knoxville, Tenn, USA, 2006.

[3] K. Abrahamyan, J. Bähr, J. P. Carneiro et al., "Experimental characterization and numerical simulations of the electron source at PITZ," Nuclear Instruments and Methods in Physics Research, Section A, vol. 558, no. 1, pp. 249-252, 2006.

[4] A. Todd, H. Bluem, V. Christina et al., "High-power electron beam injectors for $100 \mathrm{~kW}$ free-electron lasers," in Proceedings of the IEEE Particle Accelerator Conference (PAC '03), pp. 977979, May 2003.

[5] R. Nagai, R. Hajima, N. Nishimori et al., "High-voltage testing of a $500-\mathrm{kV}$ dc photocathode electron gun," Review of Scientific Instruments, vol. 81, no. 3, Article ID 033304, 5 pages, 2010.

[6] J. A. Berger, J. T. Hogan, M. J. Greco, W. A. Schroeder, A. W. Nicholls, and N. D. Browning, "DC photoelectron gun parameters for Ultrafast electron microscopy," Microscopy and Microanalysis, vol. 15, no. 4, pp. 298-313, 2009.

[7] F. Ciocci, R. Bartolini, A. Doria et al., "Operation of a compact free-electron laser in the millimeter-wave region with a bunched electron beam," Physical Review Letters, vol. 70, no. 7, pp. 928-931, 1993.
[8] M. Asakawa, N. Sakamoto, N. Inoue et al., "Experimental study of a waveguide free-electron laser using the coherent synchrotron radiation emitted from electron bunches," Applied Physics Letters, vol. 64, no. 13, pp. 1601-1603, 1994.

[9] G. Caretto, D. Doria, V. Nassisi, and M. V. Siciliano, "Photoemission studies from metal by UV lasers," Journal of Applied Physics, vol. 101, no. 7, Article ID 073109, 7 pages, 2007.

[10] P. G. O'Shea, J. A. Lancaster, R. Sachtschale, and C. R. Jones, "Radio frequency photoinjector using LaB6 cathode and a nitrogen drive laser," Applied Physics Letters, vol. 73, no. 3, pp. 411-413, 1998.

[11] M. Asakawa, K. Mima, S. Nakai, M. Fujita, K. Imasaki, and C. Yamanaka, "Use of multiphoton photoelectric process to generate a high brightness electron beam for free-electron lasers," Applied Physics Letters, vol. 64, no. 16, pp. 2056-2058, 1994. 

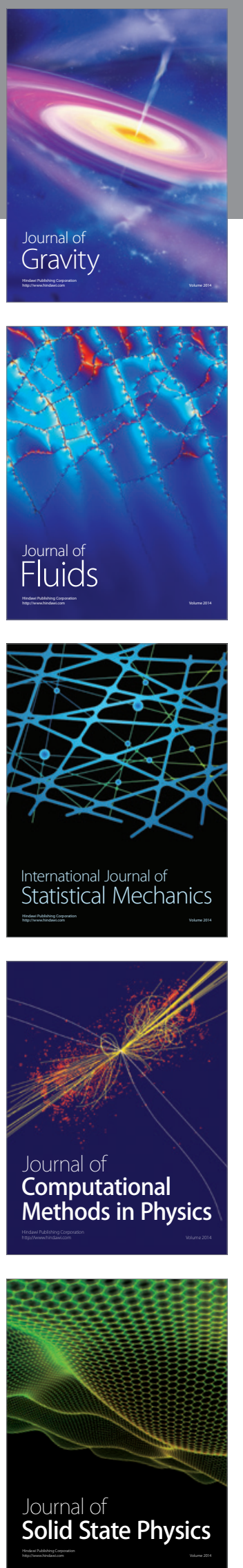

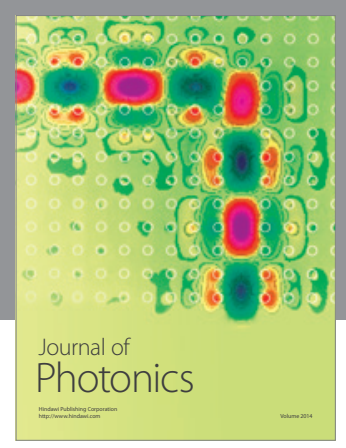

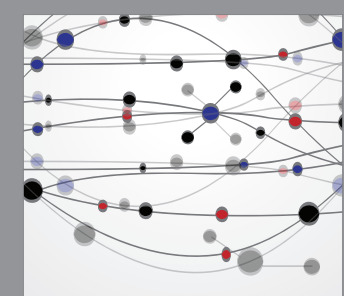

The Scientific World Journal
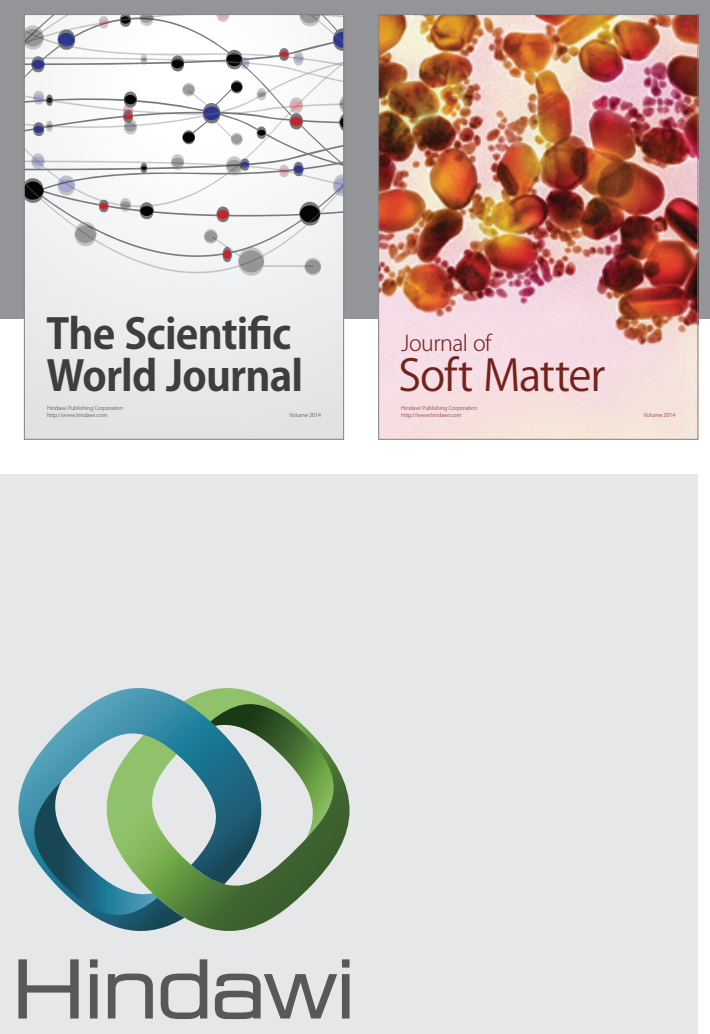

Submit your manuscripts at

http://www.hindawi.com
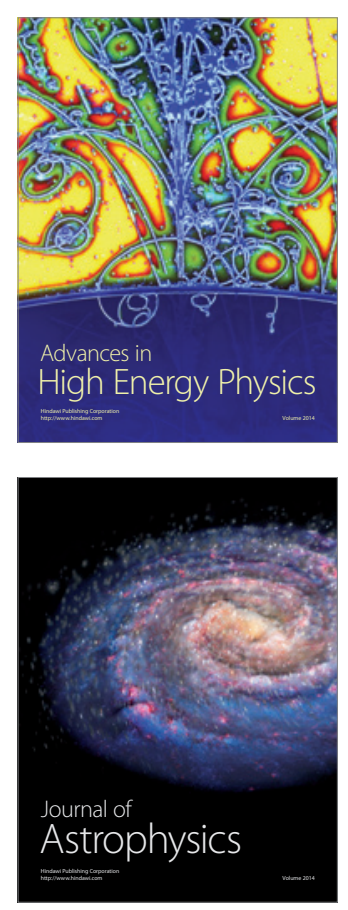
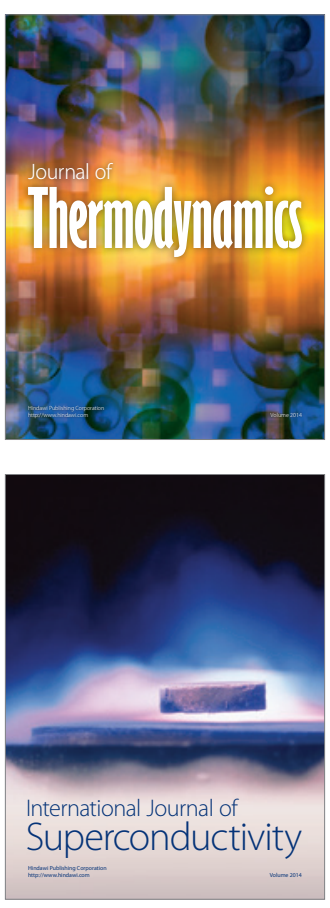
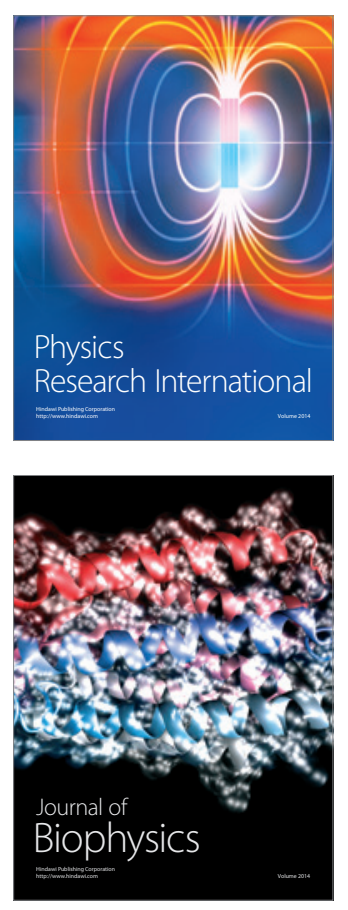
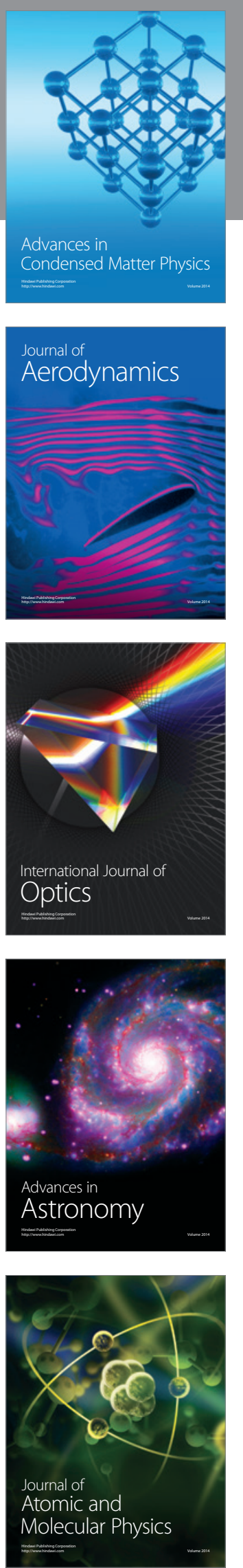